\title{
Erratum to: Data-stories about (im)patient customers in tele-queues
}

\author{
Avishai Mandelbaum • Sergey Zeltyn
}

Published online: 10 July 2013

(C) Springer Science+Business Media New York 2013

\section{Erratum to: Queueing Syst DOI 10.1007/s11134-013-9354-x}

The Acknowledgment section has been inadvertently omitted in the original publication of this article. The complete text should read as given below.

\begin{abstract}
Acknowledgments Our paper greatly benefited from a thorough and insightful refereeing process. It would not have been possible without the support of the Technion SEELab, and especially Valery Trofimov and Ella Nadjharov. The work of Avi Mandelbaum has been partially supported by BSF Grants 2005175 and 2008480, ISF Grant 1357/08 and by the Technion funds for promotion of research and sponsored research. Some of the research was funded by and carried out while Avi was visiting the Statistics and Applied Mathematical Sciences Institute (SAMSI) of the NSF; the Department of Statistics and Operations Research (STOR), the University of North Carolina at Chapel Hill; the Department of Information, Operations and Management Sciences (IOMS), Leonard N. Stern School of Business, New York University; and the Department of Statistics, The Wharton School, University of Pennsylvania-the hospitality of all these institutions is gratefully acknowledged and truly appreciated.
\end{abstract}

The online version of the original article can be found under doi:10.1007/s11134-013-9354-X.

A. Mandelbaum

Faculty of Industrial Engineering \& Management, Technion, 32000 Haifa, Israel

e-mail: avim@ie.technion.ac.il

\section{S. Zeltyn ( $₫)$}

IBM Research Lab, 31905 Haifa, Israel

e-mail: sergeyz@il.ibm.com 